\title{
The inhibitory mechanism of Cordyceps sinensis on cigarette smoke extract-induced senescence in human bronchial epithelial cells
}

This article was published in the following Dove Press journal:

International Journal of COPD

28 July 2016

Number of times this article has been viewed

\author{
Ailing Liu ${ }^{1,2, *}$ \\ Jinxiang $\mathrm{Wu}^{1, *}$ \\ Aijun $\mathrm{Li}^{2}$ \\ Wenxiang $\mathrm{Bi}^{3}$ \\ Tian Liu' \\ Liuzhao Cao' \\ Yahui Liu' \\ Liang Dong'
}

'Department of Pulmonary Diseases, Qilu Hospital, Shandong University, Jinan, Shandong, People's Republic of China; ${ }^{2}$ Department of Pulmonary Diseases, Weihai Municipal Hospital, Weihai, Shandong, People's Republic of China; ${ }^{3}$ Institute of Biochemistry and Molecular Biology, School of Medicine, Shandong University, Jinan, Shandong, People's Republic of China

*These authors contributed equally to this work
Correspondence: Liang Dong Department of Pulmonary Diseases, Qilu Hospital, Shandong University, \#107, Wenhua Xi Road, Jinan City 25002I, Shandong Province, People's Republic of China Email d15506@I26.com
Objectives: Cellular senescence is a state of irreversible growth arrest induced either by telomere shortening (replicative senescence) or stress. The bronchial epithelial cell is often injured by inhaled toxic substances, such as cigarette smoke. In the present study, we investigated whether exposure to cigarette smoke extract (CSE) induces senescence of bronchial epithelial cells; and Cordyceps sinensis mechanism of inhibition of CSE-induced cellular senescence.

Methods: Human bronchial epithelial cells (16HBE cells) cultured in vitro were treated with CSE and/or C. sinensis. p16, p21, and senescence-associated-galactosidase activity were used to detect cellular senescence with immunofluorescence, quantitative polymerase chain reaction, and Western blotting. Reactive oxygen species (ROS), PI3K/AKT/mTOR and their phosphorylated proteins were examined to testify the activation of signaling pathway by ROS fluorescent staining and Western blotting. Then, inhibitors of ROS and PI3K were used to further confirm the function of this pathway.

Results: Cellular senescence was upregulated by CSE treatment, and $C$. sinensis can decrease CSE-induced cellular senescence. Activation of ROS/PI3K/AKT/mTOR signaling pathway was enhanced by CSE treatment, and decreased when C. sinensis was added. Blocking ROS/PI3K/ AKT/mTOR signaling pathway can attenuate CSE-induced cellular senescence.

Conclusion: CSE can induce cellular senescence in human bronchial epithelial cells, and ROS/ $\mathrm{PI} 3 \mathrm{~K} / \mathrm{AKT} / \mathrm{mTOR}$ signaling pathway may play an important role in this process. $C$. sinensis can inhibit the CSE-induced senescence.

Keywords: senescence, Cordyceps sinensis, ROS/PI3K/AKT/mTOR signaling pathway, COPD

\section{Introduction}

Chronic obstructive pulmonary disease (COPD) is a progressive disabling illness associated with an abnormal inflammatory response of the airways and the lungs to noxious stimuli. It is characterized by persistent airflow limitation that is not fully reversible and airway inflammation. ${ }^{1}$ Recently, it has been shown that cellular senescence might play a role in the pathogenesis of COPD. ${ }^{2}$

Cellular senescence is a process that results from a variety of stresses, leading to a state of irreversible growth arrest. Senescent cells accumulate during aging and have been implicated in promoting a variety of age-related diseases, such as ischemic heart disease, Alzheimer's disease, and COPD. ${ }^{3}$

Telomere shortening, activation of PI3K-AKT-mTOR signaling pathway, impaired autophagy, mitochondrial dysfunction, stem cell exhaustion, and low grade chronic inflammation are the main mechanisms of cell senescence. ${ }^{4}$ Many of these pathways are driven by chronic oxidative stress. Increased oxidative stress is well documented 
in the lungs of patients with COPD. This increase in oxidative stress is due to oxidants in cigarette smoke and activated inflammatory cells. Reactive oxygen species (ROS) are generated in oxidative stress, and activate PI3K, then, via activation of the kinase AKT, activates mTOR, the PI3K-AKT-mTOR signaling pathway is activated. Then, the activated pathway inhibits autophagy via unc-51-like autophagy activating kinase- 1 and reduces sirtuin- 1 activity, both of which accelerate the aging process. ${ }^{5}$ The pathway of mTOR is a key modulator of aging and age-related disease. ${ }^{6}$ Activation of the mTOR pathway may play an important role in multimorbidity, and so inhibition of this pathway may offer a good future therapeutic opportunity. ${ }^{7}$

In the People's Republic of China, many traditional Chinese medicines modalities are regularly used in the treatment of patients with COPD. Several clinical trials ${ }^{8,9}$ have shown that traditional Chinese medicines might have a therapeutic effect on patients with COPD, including improvement of symptoms, quantity of life, and lung function. Cordyceps sinensis, or Dong Chong Xia Cao in Chinese, is a special type of medicinal mushroom, which forms on an insect larva infected by the $C$. sinensis fungus; it is rich in nucleoside, polysaccharide, sterol, protein, amino acid, and polypeptide. ${ }^{10}$ Now research on $C$. sinensis is mainly focused on chemical constituents and pharmacological actions. ${ }^{11}$ C. sinensis has the functions of antiaging, anti-inflammatory, antioxidant, antitumor, antiapoptosis, and can regulate endocrine, respiratory, immune, and nervous systems. ${ }^{12}$ But the specific inhibitory mechanism of $C$. sinensis is not clear. Singh et $\mathrm{al}^{13}$ found $C$. sinensis could decrease oxidative stress in human lung epithelial cells. Antioxidation plays an important role in the functions of $C$. sinensis. The oxidative stress in the respiratory system is mainly caused by smoke, which has a close association with cell senescence. ${ }^{14}$

Based on earlier research mentioned in the previous paragraph, the functions of $C$. sinensis are wide, but there is no definite research about its effect on cell senescence and specific cellular mechanisms. Here, we investigate the inhibitory effect of $C$. sinensis on the senescence of human bronchial epithelial cells induced by cigarette smoke extract (CSE) and its mechanism.

\section{Materials and methods}

\section{Cells and regents}

Ethical approval was not required by the institutional review board of Qilu Hospital, Shandong University, because the cells mentioned in the experiment were derived from cell lines. The human bronchial epithelial cell line, 16HBE, was purchased from a cell bank (ATCC, Manassas, VA, USA) and cultured in high glucose Dulbecco's Modified Eagle's Medium (H-DMEM) complete medium supplemented with $10 \%$ fetal bovine serum (FBS), $2 \mathrm{mM}$ glutamine, $100 \mathrm{U} / \mathrm{mL}$ of penicillin, and $100 \mu \mathrm{g} / \mathrm{mL}$ of streptomycin at $37^{\circ} \mathrm{C}$ under conditions of $5 \% \mathrm{CO}_{2}$. After 2 days in culture, the adherent cells were consistently $50 \%$ of epithelial morphology. The cells were treated with CSE and/or C. sinensis (2 hours before adding CSE).

CSE was prepared by a modification of the method of Carp and Janoff; briefly, three cigarettes without filters were combusted in a modified gas collecting pipe. ${ }^{15}$ The smoke was bubbled through $3 \mathrm{~mL}$ of phosphate-buffered saline. The resulting suspension was adjusted to $\mathrm{pH} 7.4$ with concentrated $\mathrm{NaOH}$ and then filtered through a $0.22 \mu \mathrm{m}$ pore filter (MILLEX ${ }^{\circledR} \mathrm{GP}$ ) to remove bacteria and large particles. CSE was applied to $16 \mathrm{HBE}$ cultures within 30 minutes of preparation. To make sure the concentration of CSE was stable, the burning time and the pressure of gas collecting pipe were fixed. The initial absorbance value was determined in the range of CSE (270-280 nm) by using the spectrophotometer, and the absorbance value of CSE was the same as that for each preparation. CSE solution was diluted by adding H-DMEM containing $10 \%$ FBS to concentrations of $0.5 \%$, $1 \%, 2 \%$, and $5 \%$.

Cultured $C$. sinensis extracts were provided by Hangzhou Zhongmei Huadong Pharmaceutical Co Ltd. (Hangzhou, People's Republic of China) at a concentration of $0.99 \mathrm{~g} / \mathrm{mL}$; it was microfiltered to remove bacteria. C. sinensis was diluted by adding H-DMEM containing $10 \%$ FBS to a concentration of $100 \mathrm{mg} / \mathrm{L} .{ }^{16,17}$

The PI3K signaling pathway inhibitor Ly294002 (\#9901, Cell Signaling Technology, Danvers, MA, USA) $10 \mu \mathrm{M}^{18}$ and ROS inhibitor $N$-acetylcysteine (S0077, Beyotime, Haimen, People's Republic of China) 10 mM were added. ${ }^{19}$

\section{3-(4, 5-Dimethylthiazol-2-yl)-2, 5-diphenyltetrazolium bromide assay}

$16 \mathrm{HBE}$ cells plated in 96-well plates were treated with $\mathrm{CSE}$ in different doses and time points. Cell viability was assayed using the Vibrant ${ }^{\circledR}$ MTT Cell Proliferation Assay Kit (Molecular Probes, Thermo Fisher Scientific, Waltham, MA, USA) following the manufacturer's protocol.

\section{Staining for senescence-associated- $\beta$-galactosidase}

$16 \mathrm{HBE}$ cells were fixed in $2 \%$ formaldehyde and $0.2 \%$ glutaraldehyde for 10 minutes at room temperature, washed in phosphate-buffered saline, and incubated for 24 hours at $37^{\circ} \mathrm{C}$ with senescence-associated $\beta$-galactosidase (SA- $\beta$-gal) 
(Sigma-Aldrich, St Louis, MO, USA) in staining solution (40 mM sodium citrate [pH 6], $150 \mathrm{mM} \mathrm{NaCl}, 5 \mathrm{mM}$ potassium ferricyanide, $5 \mathrm{mM}$ potassium ferrocyanide, $2 \mathrm{mM} \mathrm{MgCl}_{2}$, and $1 \mathrm{mg} / \mathrm{mL}$ bromo-4-chloro-3-indolyl- $\beta$-D-galactoside).

\section{Western blotting}

$16 \mathrm{HBE}$ cells were grown to confluence in six-well plates. After treatment with CSE, C. sinensis, N-acetylcysteine, or LY294002, 16HBE cells were lysed with a cell extraction buffer (Thermo Fisher Scientific), and the total amount of protein was quantitated using the Pierce BCA Protein Assay Kit (P0010S, Beyotime). Equal amounts of total protein (30 $\mu \mathrm{g}$ ) were separated on $10 \%-12 \%$ Bis-Tris gels in MOPS SDS Running Buffer (Thermo Fisher Scientific), transferred to a polyvinylidene difluoride membrane (Immobilon, Millipore Corp, Bedford, MA, USA).

Then the polyvinylidene difluoride membrane was blocked with 5\% skimmed milk in Tris-buffered saline (50 mM Tris- $\mathrm{Cl}, \mathrm{pH} 7.5,150 \mathrm{mM} \mathrm{NaCl}$ ) for 60 minutes at room temperature and probed with anti-p16 antibody (1:2,000; Abcam, Cambridge, MA, USA), anti-p21 antibody (1:500; Abcam), anti-PI3K antibody (1:500; Cell Signaling Technology), anti-AKT antibody $(1: 2,000$; Cell Signaling Technology), anti-p-AKT antibody (1:1,000; Cell Signaling Technology), anti-mTOR antibody $(1: 1,000$; Cell Signaling Technology), and anti-p-mTOR antibody (1:1,000; Cell Signaling Technology) followed by overnight incubation with primary antibodies. The membrane was washed three times with $1 \times$ TBS-T for 5 minutes and then incubated for 1 hour with secondary antibodies conjugated to horseradish peroxidase at room temperature. The membrane was exposed to a multicolor fluorescence gel imaging analysis system (FluorChem ${ }^{\circledR} \mathrm{Q}$, Cell Biosciences, Santa Clara, CA, USA) and visualized using the ECL system (Santa Cruz Biotechnology, Santa Cruz, CA, USA). The integrated density value of band intensities from the films was analyzed by the ImageJ software (National Institutes of Health, Bethesda, MA, USA).

\section{Real-time quantitative polymerase chain reaction analysis ${ }^{20}$}

$16 \mathrm{HBE}$ cells were grown to confluence in six-well plates. After transfection with CSE and/or $C$. sinensis extract, RNA was isolated using TRIzol (Thermo Fisher Scientific), and quantified using a NanoDrop (Thermo Fisher Scientific). RNA was analyzed by real-time polymerase chain reaction (PCR) amplification. Briefly, $1 \mu \mathrm{g}$ of total RNA per sample was denaturated at $70^{\circ} \mathrm{C}$ for 10 minutes and laid on ice for 10 minutes, PCR reactions were performed in a volume of $20 \mu \mathrm{L}$ containing $4 \mu \mathrm{L} 5 \times$ reverse transcriptase $(\mathrm{RT})$ buffer
(Toyobo, Osaka, Japan), $1 \mu \mathrm{L}$ RT Enzyme Mix (Toyobo), $1.0 \mu \mathrm{L}$ ( $5 \mathrm{pmol}$ ) of each primer (sense and antisense) in the presence of PCR buffer (Toyobo). The complementary DNAs (cDNAs) were predenaturated for 2 minutes at $95^{\circ} \mathrm{C}$ followed by 35 cycles of 30 seconds denaturation at $95^{\circ} \mathrm{C}, 30$ seconds annealing at $60^{\circ} \mathrm{C}$, and 1 minute elongation at $68^{\circ} \mathrm{C}$.

p16 was amplified by using the following primers (157 bp): forward primer $\left(5^{\prime}-3^{\prime}\right)$ : CTACTCTCCTCCGCTGGGAA and reverse primer (5'-3'): GGCCTAACTTAGCGCTGCTT. p21 was amplified by using the following primers (74 bp): forward primer $\left(5^{\prime}-3^{\prime}\right)$ : 5'-CAGGCTCAGGAGTTAGCAAGG and reverse primer $\left(5^{\prime}-3^{\prime}\right)$ : TCAACACCCTGTCTTGTCTTCG. Glyceraldehyde 3-phosphate dehydrogenase was amplified by using the following primers ( $89 \mathrm{bp}$ ): forward primer $\left(5^{\prime}-3^{\prime}\right)$ : ATGATTCATCCCACGGCAAG and reverse primer $\left(5^{\prime}-3^{\prime}\right)$ : CTGGAAGATGGTGATGGGTT.

Real-time PCR reactions were performed in a volume of $20 \mu \mathrm{L}$ containing $2 \mu \mathrm{L}$ of cDNA, $8 \mu \mathrm{L}$ of each primer $(10 \mathrm{pmol} / \mu \mathrm{L}, 10 \mu \mathrm{M})$ and $10 \mu \mathrm{L}$ of QuantiTect ${ }^{\mathrm{TM}}$ SYBRs Green PCR containing DNA polymerase, dNTP mix, buffer, $\mathrm{MgCl}_{2}$, and fluorescent dyes (Qiagen, Mississauga, Ontario, Canada). The PCR protocol consisted of three programs: denaturation, amplification, and melting curve analysis for product identification. The denaturation and amplification conditions were $95^{\circ} \mathrm{C}$ for 20 minutes followed by 40 cycles of PCR. Each cycle included denaturation at $95^{\circ} \mathrm{C}$ for 30 seconds, annealing of 10 seconds at $60^{\circ} \mathrm{C}$, and extension of 15 seconds at $72^{\circ} \mathrm{C}$. The primers of $\mathrm{p} 16, \mathrm{p} 21$, and glyceraldehyde 3-phosphate dehydrogenase were stated as above. The temperature transition rate was $20^{\circ} \mathrm{C} / \mathrm{s}$, except when heating at $72^{\circ} \mathrm{C}$, when it was $5^{\circ} \mathrm{C} / \mathrm{s}$. Fluorescence was measured at the end of every cycle to allow quantification of cDNA. Data were acquired and analyzed with the computer software Opticon Monitor (version 2.02.24; BioRad Laboratories, Hercules, CA, USA). Normalized expressions were calculated by using the calculated efficiency of each PCR reaction with a cDNA standard curve.

\section{Immunofluorescence microscopy ${ }^{21}$}

$16 \mathrm{HBE}$ cells cultured on glass slides were fixed with 4\% paraformaldehyde for 10 minutes, incubated with $0.5 \%$ Triton $\mathrm{X}-100$ for 30 minutes, and then blocked with $1 \%$ bovine serum albumin for 30 minutes at room temperature. The slides were incubated with anti-p16 (1:50) and anti-p21 (1:200) overnight at $4{ }^{\circ} \mathrm{C}$ and subsequently with goat anti-rabbit immunoglobulin G-fluorescein isothiocyanate mAb (1:200) for 1 hour. 4',6-Diamidino-2-phenylindole (500 ng/mL in 95\% ethanol) was used to stain the nuclei for 20 seconds. Cover slips were mounted with $80 \%$ glycerol (Zsbio, Beijing, People's 
Republic of China). The slides were examined under fluorescence microscopy (Nikon, Gotemba, Japan).

\section{ROS}

ROS assay kit (E004, Nanjing Jiangcheng Bioengineering Institute, Nanjing, People's Republic of China) was used to detect intracellular ROS level following the manufacturer's protocol. 4',6-Diamidino-2-phenylindole (500 ng/mL in 95\% ethanol) was used to stain the nuclei for 20 seconds. Stained cells were observed with fluorescence microscope (Nikon).

\section{Statistical analysis}

All data were from at least three separate experiments for Western blotting and ten separate experiments for MTT and staining for SA- $\beta$-gal. Statistical analysis was performed using the SPSS 12.0 software package (SPSS Inc., Chicago, IL, USA). Data were analyzed using one-way analysis of variance between the groups studied. Significance was defined for $P<0.05$.

\section{Results}

\section{CSE-induced cellular senescence in human bronchial epithelial cells in vitro}

To detect the appropriate dose and action time of CSE, the cell survival rate was observed by MTT assays. 16HBE cells were treated with CSE at different doses and time points. The relative cell number was detected to evaluate cell growth. Cell survival rate was inhibited by CSE in a time- and dose-dependent manner. According to the survival curve (Figure 1), considering $\mathrm{IC}_{50}$ and the obvious downward trend, stimulation by $2 \%$ CSE for 24 hours could be used in the follow-up experiments.

\section{C. sinensis can inhibit the CSE-induced cellular senescence}

Previous reports have demonstrated that cigarette smoke induces bronchial epithelial cellular senescence, ${ }^{22}$ and senescent cells are readily found in airway epithelia of patients with COPD. ${ }^{23}$ Two major signaling pathways are involved in senescence: the $\mathrm{p} 19 \mathrm{ARF} / \mathrm{p} 53 / \mathrm{p} 21$ and the $\mathrm{p} 16 \mathrm{INK} 4 / \mathrm{CDK} /$ pRb pathways. ${ }^{24}$ We examined p21 and p16 protein levels to evaluate cell senescence, and higher $\mathrm{p} 16$ and $\mathrm{p} 21$ protein levels were found in epithelial cells in a time- and dosedependent manner after CSE treatment (Figure 2A and B). However, higher p16 and p21 expressions induced by CSE could be reduced when treated with $C$. sinensis (Figure $3 \mathrm{~A}$ ). Immunofluorescence cytochemistry and messenger RNA levels of p16 and p21 were also analyzed to verify the result. They were significantly increased in $16 \mathrm{HBE}$ cells after CSE

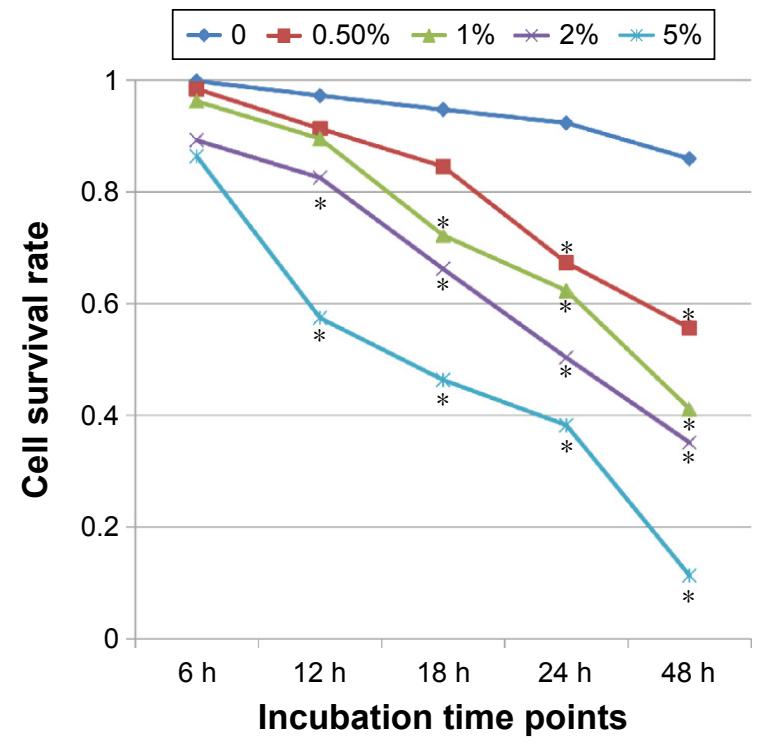

Figure I I6HBE cells were incubated with CSE at different doses and time points. Notes: Cell survival rate was assayed using the MTT assays to evaluate cell viability $(* P<0.05$, vs control at respective time points). Data are expressed as mean $\pm S E$. Results represent three independent experiments.

Abbreviations: CSE, cigarette smoke extract; HBE, human bronchial epithelial; MTT, 3-(4, 5-dimethylthiazol-2-yl)-2, 5-diphenyltetrazoliumbromide; SE, standard error.

stimulation, but decreased when the cells were treated with C. sinensis before CSE stimulation (Figure 3C and D). SA- $\beta$-gal staining was performed to examine senescent cells. $\beta$-Galactosidase, the lysosomal hydrolase, is active at $\mathrm{pH} 4$, but $\mathrm{SA}-\beta$-gal is active at $\mathrm{pH} 6$ and is only present in senescent cells; allowing the two activities to be readily distinguished. ${ }^{25} \mathrm{We}$ found SA- $\beta$-gal positive cells ratio was obviously increased by CSE stimulation. After adding C. sinensis, the ratio was able to be decreased compared to CSE group (Figure 3B). These data indicated that CSE stimulation could induce cellular senescence in human bronchial epithelial cells, and $C$. sinensis can inhibit the senescence induced by CSE.

\section{The role of ROS/mTOR signaling pathway and C. sinensis in CSE-induced senescence} ROS and PI3K/AKT/mTOR signaling pathway are involved in a variety of physiological processes, including cellular senescence. ${ }^{26}$ According to previous studies, ${ }^{26}$ we intend to determine the importance of ROS/mTOR signaling pathway in cellular senescence and the correlation between each other in this process. ROS can be caused by smoke, we used CSE to stimulate $16 \mathrm{HBE}$ cells and detect the change of ROS and the activation of PI3K/AKT/mTOR signaling pathway. We found ROS fluorescence was enhanced by CSE, and $C$. sinensis can weaken the ROS change (Figure 4). The expressions of p-AKT and p-mTOR were promoted by CSE in a time- and dose-dependent manner (Figure 5A and B). Activation of 


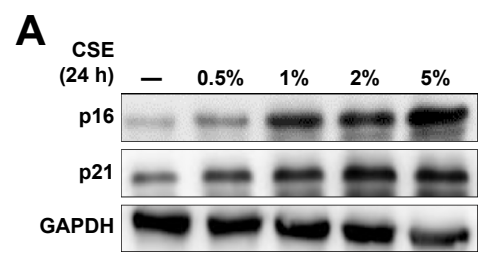

B

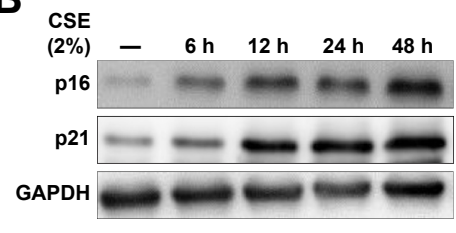

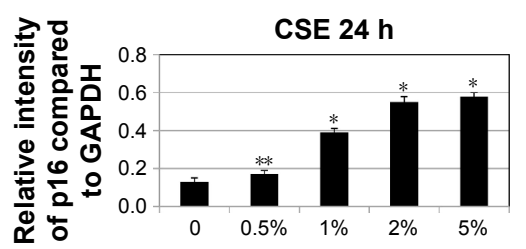
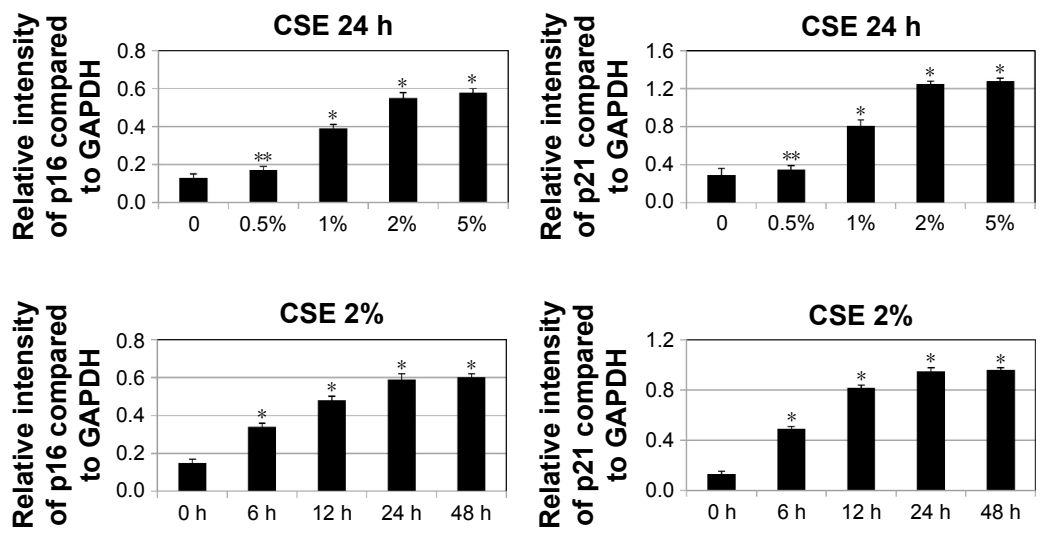

Figure 2 Cellular senescence induced by CSE stimulation.

Notes: (A) 16HBE cells were stimulated with different doses of CSE for 24 hours, protein expressions of pl6 and $\mathrm{p} 2 \mathrm{I}$ were detected by Western blotting ( $* \mathrm{P}<0.05$, $* * P>0.05$ ). (B) $16 \mathrm{HBE}$ cells were stimulated by $2 \%$ CSE for different time durations and protein expressions of p 16 and $p 21$ were detected by Western blotting ( $* P<0.05$ ). Data are expressed as mean $\pm \mathrm{SE}$. Results represent at least three independent experiments.

Abbreviations: CSE, cigarette smoke extract; GAPDH, glyceraldehyde 3-phosphate dehydrogenase; HBE, human bronchial epithelial; SE, standard error.

A

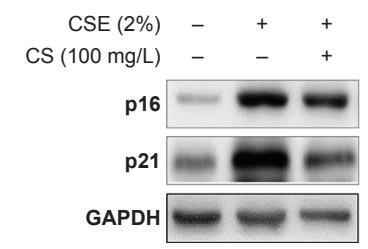

B CSE $(2 \%)$ CS (100 mg/L)

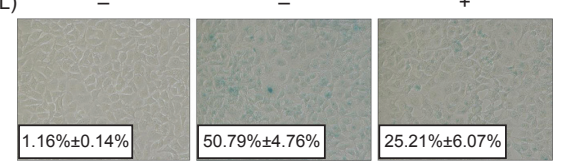

(200x)

C $\begin{array}{r}\operatorname{cse}(2 \%) \\ \operatorname{Cs}(100 \mathrm{mg} / \mathrm{L})\end{array}$
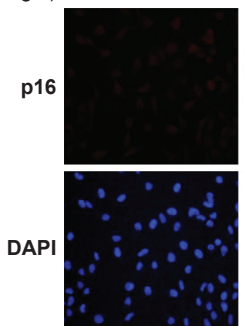

D
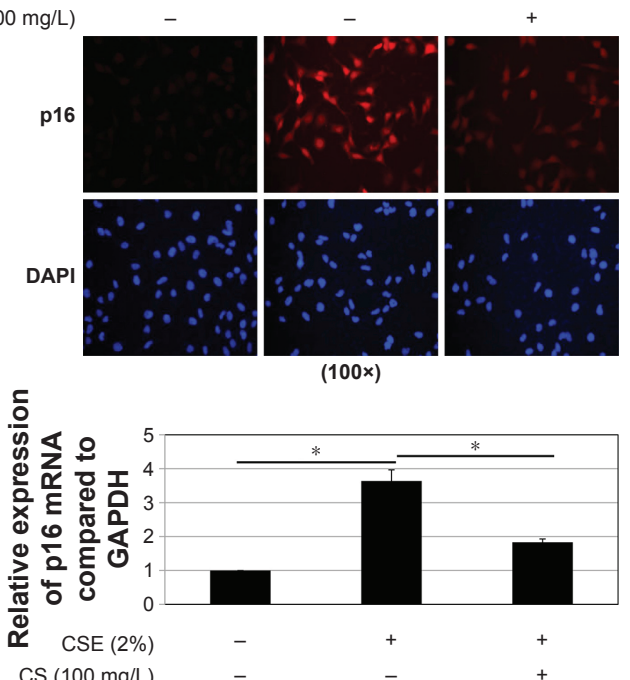
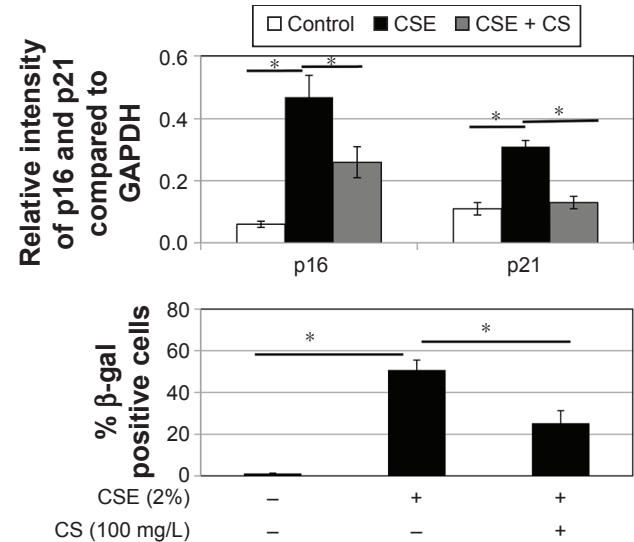

CSE $(2 \%)$ CS (100 mg/L)
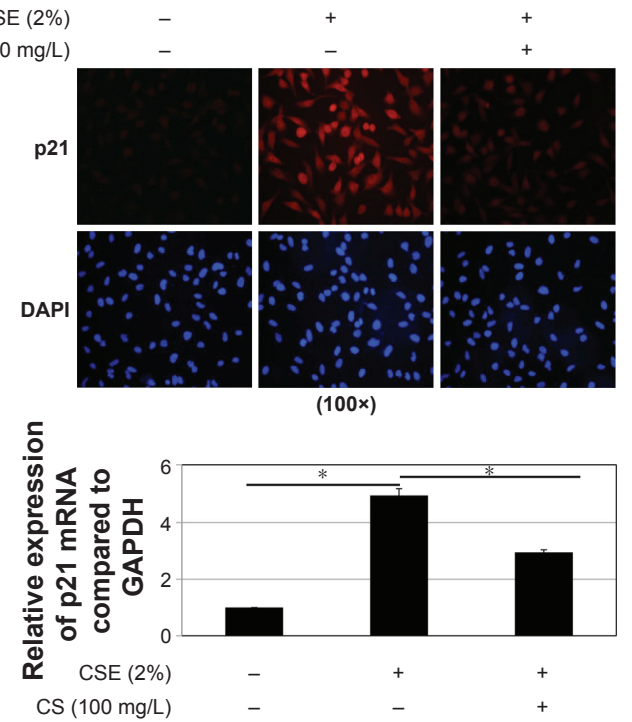

Figure 3 Cellular senescence affected by CSE and Cordyceps sinensis. I6HBE cells were stimulated with $2 \%$ CSE and/or C. sinensis (I00 mg/L) ( 2 hours before adding CSE) for 24 hours.

Notes: (A) The protein expressions of p16 and p2I were detected by Western blotting $(* P<0.05)$. (B) The senescent cells in CSE and CSE $+C$. sinensis groups were examined by SA- $\beta$-gal staining. SA- $\beta$-gal positive cells were enumerated by counting over 400 cells in three independent fields (*P $<0.05$ ). (C) The expression of $p / 6$ and p2I in CSE and CSE $+C$. sinensis groups was determined by immunofluorescence cytochemistry. (D) The expression of pl6 and p2I in CSE and CSE + C. sinensis groups was detected by $q P C R(* P<0.05)$. Data are expressed as mean $\pm S E$. Results represent at least three independent experiments.

Abbreviations: CS, Cordyceps sinensis; CSE, cigarette smoke extract; DAPI, 4',6-diamidino-2-phenylindole; GAPDH, glyceraldehyde 3-phosphate dehydrogenase; HBE, human bronchial epithelial; mRNA, messenger RNA; qPCR, quantitative polymerase chain reaction; SA- $\beta$-gal, senescence-associated $\beta$-galactosidase; SE, standard error. 

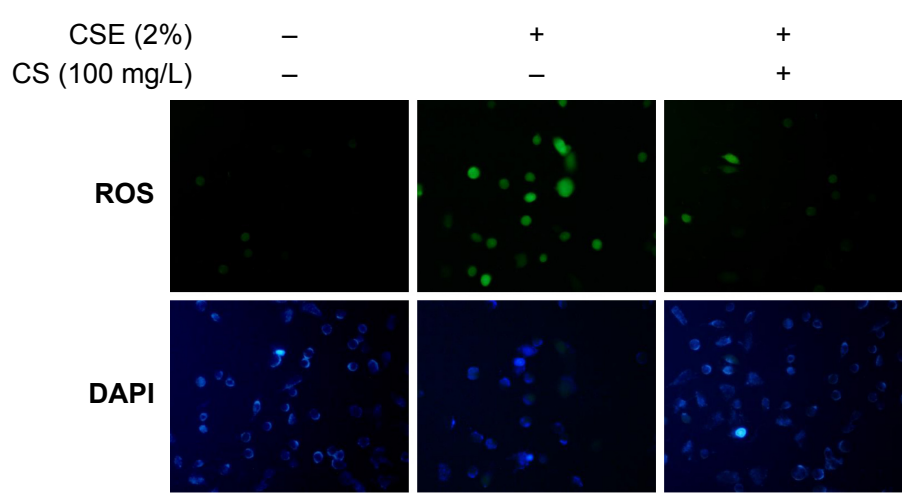

$(200 x)$

Figure 4 ROS fluorescence in I6HBE cells.

Notes: I6HBE cells were stimulated with $2 \%$ CSE and/or Cordyceps sinensis ( $100 \mathrm{mg} / \mathrm{L})$ for 24 hours, and stained with ROS fluorescence. Results represent three independent experiments.

Abbreviations: CSE, cigarette smoke extract; DAPI, 4',6-diamidino-2-phenylindole; HBE, human bronchial epithelial; ROS, reactive oxygen species.

mTOR signaling pathway was increased in the CSE group, and decreased when C. sinensis was added (Figure 5C).

As we discussed earlier, CSE can induce cellular senescence, and C. sinensis was able to block the progress. ROS generation and ROS/mTOR signaling pathway play important roles in CSE-induced cellular senescence.
Blocking ROS/mTOR signaling pathway can alleviate CSE-induced cellular senescence in human bronchial epithelial cell

To confirm these findings, p16 and p21 expression analysis and SA- $\beta$-gal staining were also performed. As shown in
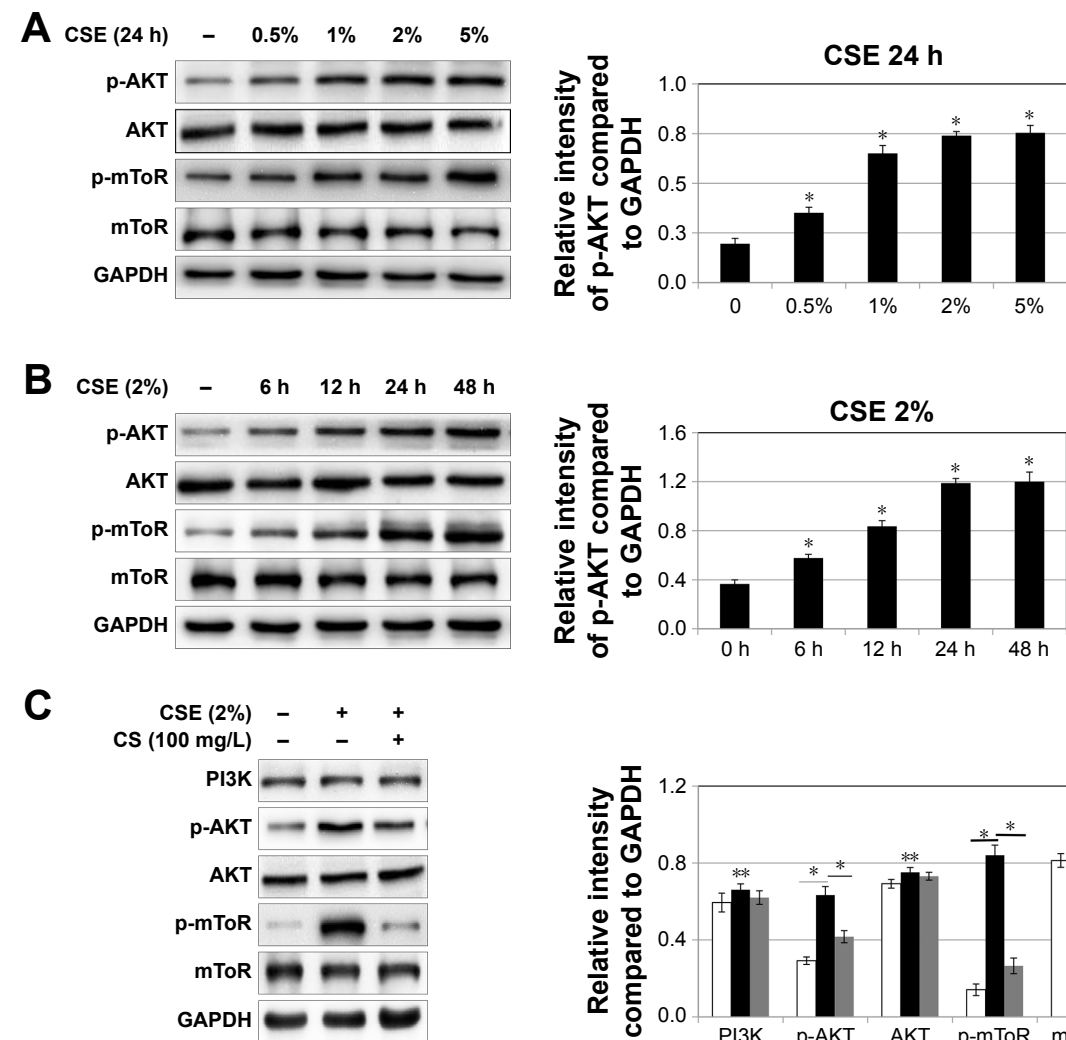
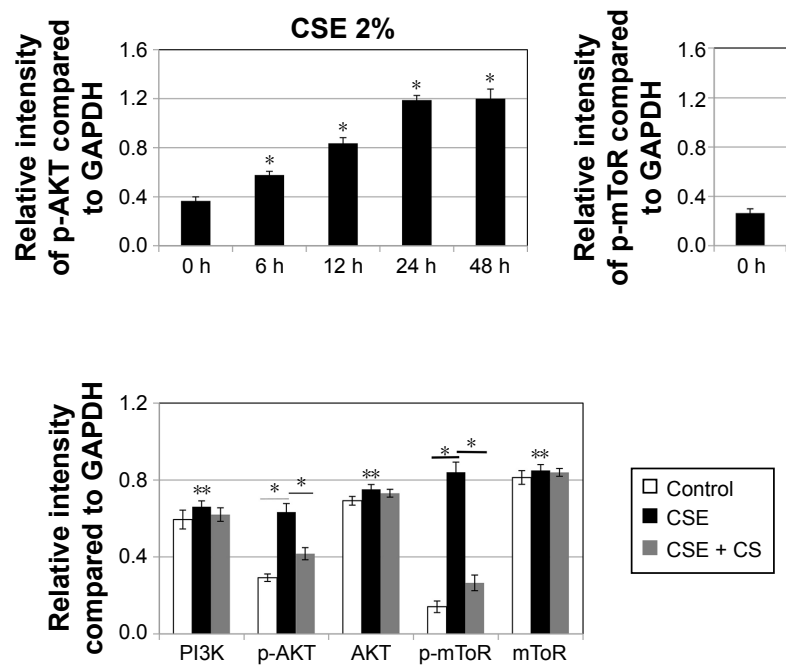

Figure 5 Expressions of PI3K, AKT, p-AKT, mTOR, p-mTOR in I6HBE cells.

Notes: (A) 16HBE cells were stimulated with different doses of CSE for 24 hours, cells were collected and total proteins were extracted and analyzed by Western blotting $(* P<0.05)$. (B) Then I6HBE cells were stimulated with $2 \%$ CSE for different time durations, total proteins were extracted and analyzed $(* P<0.05)$. (C) I6HBE cells were stimulated with $2 \%$ CSE or/and Cordyceps sinensis ( $100 \mathrm{mg} / \mathrm{L})$ for 24 hours, total proteins were extracted and analyzed by Western blotting $(* P<0.05$, $* * P>0.05)$. Data are expressed as mean \pm SE. Results represent three independent experiments.

Abbreviations: CS, Cordyceps sinensis; CSE, cigarette smoke extract; GAPDH, glyceraldehyde 3-phosphate dehydrogenase; HBE, human bronchial epithelial; mTOR, mammalian target of rapamycin; $\mathrm{PI}$ K, phosphoinositide-3-kinase; SE, standard error. 


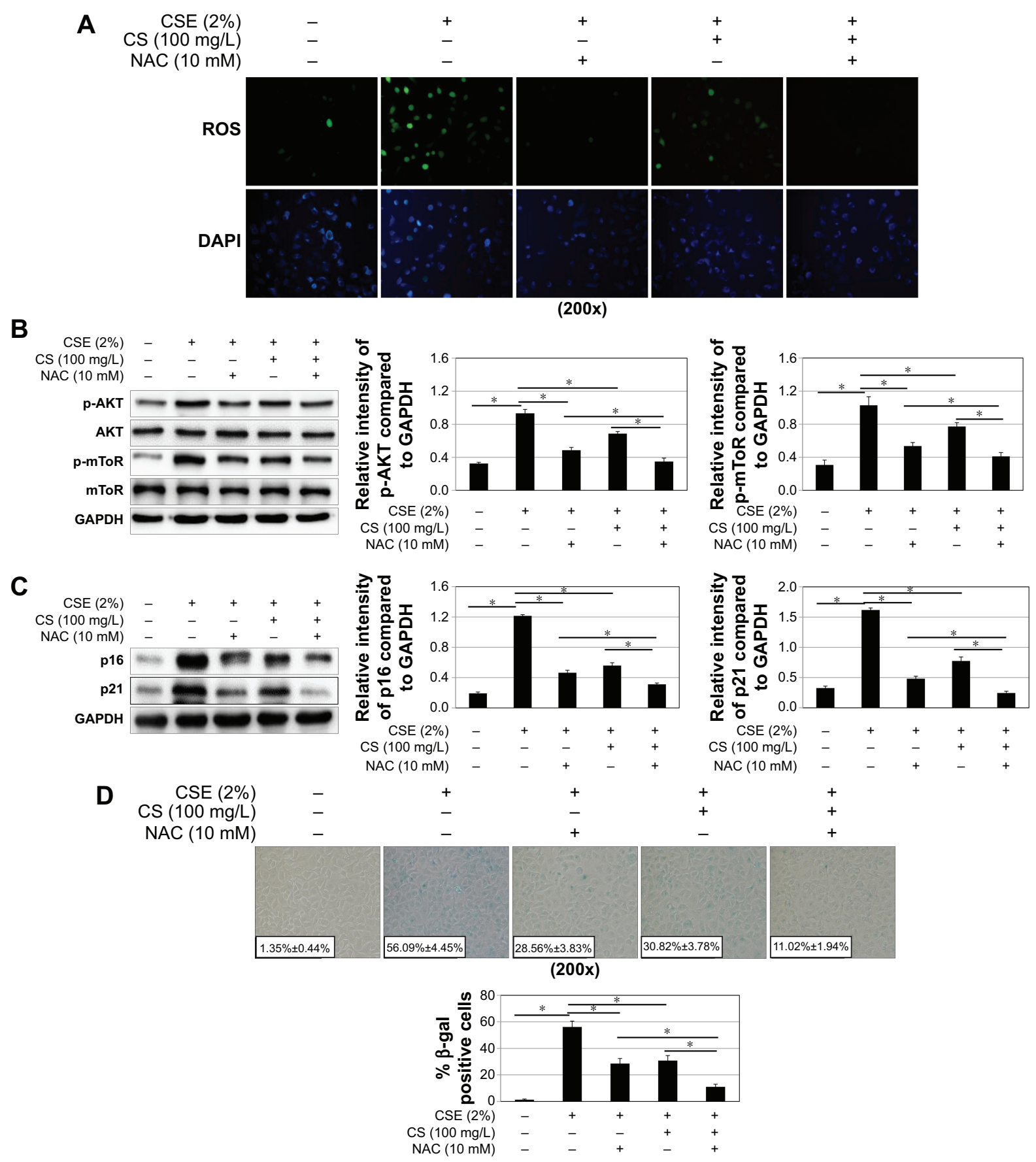

Figure 6 The effect of mTOR pathway activation and cellular senescence by blocking ROS. 16HBE cells were stimulated with 2\% CSE, $100 \mathrm{mg} / \mathrm{L}$ Cordyceps sinensis or/and $10 \mathrm{mMNAC}$.

Notes: (A) I6HBE cells were stained with ROS fluorescence. (B) Total protein was collected to detect the expressions of AKT, p-AKT, mTOR, and p-mTOR by Western blotting $(* P<0.05)$. (C) The protein expressions of p16 and p2I were detected by Western blotting $\left({ }^{*} P<0.05\right)$. (D) The senescent cells were examined by SA- $\beta$-gal staining $(* P<0.05)$. Data are expressed as mean $\pm S E$. Results represent at least three independent experiments.

Abbreviations: CS, Cordyceps sinensis; CSE, cigarette smoke extract; DAPI, 4'6-diamidino-2-phenylindole; GAPDH, glyceraldehyde 3-phosphate dehydrogenase; HBE, human bronchial epithelial; mTOR, mammalian target of rapamycin; NAC, N-acetylcysteine; SA- $\beta$-gal, senescence-associated $\beta$-galactosidase; ROS, reactive oxygen species; $\mathrm{SE}$, standard error.

Figure 6A, C, and D, we inhibited ROS with N-acetylcysteine, and ROS generation and cellular senescence induced by CSE was weakened. Activation of mTOR signaling pathway was decreased (Figure 6B).

Next, we inhibited PI3K with LY294002. The cellular senescence induced by CSE was decreased, same as earlier
(Figure $7 \mathrm{C}$ and $\mathrm{D}$ ). The activation of mTOR signaling pathway was decreased (Figure 7B). However, there was no change in ROS generation (Figure 7A).

Our study suggested that CSE can induce human bronchial epithelial cell senescence via ROS and mTOR signaling pathway. In this process, ROS generated from CSE, activates 


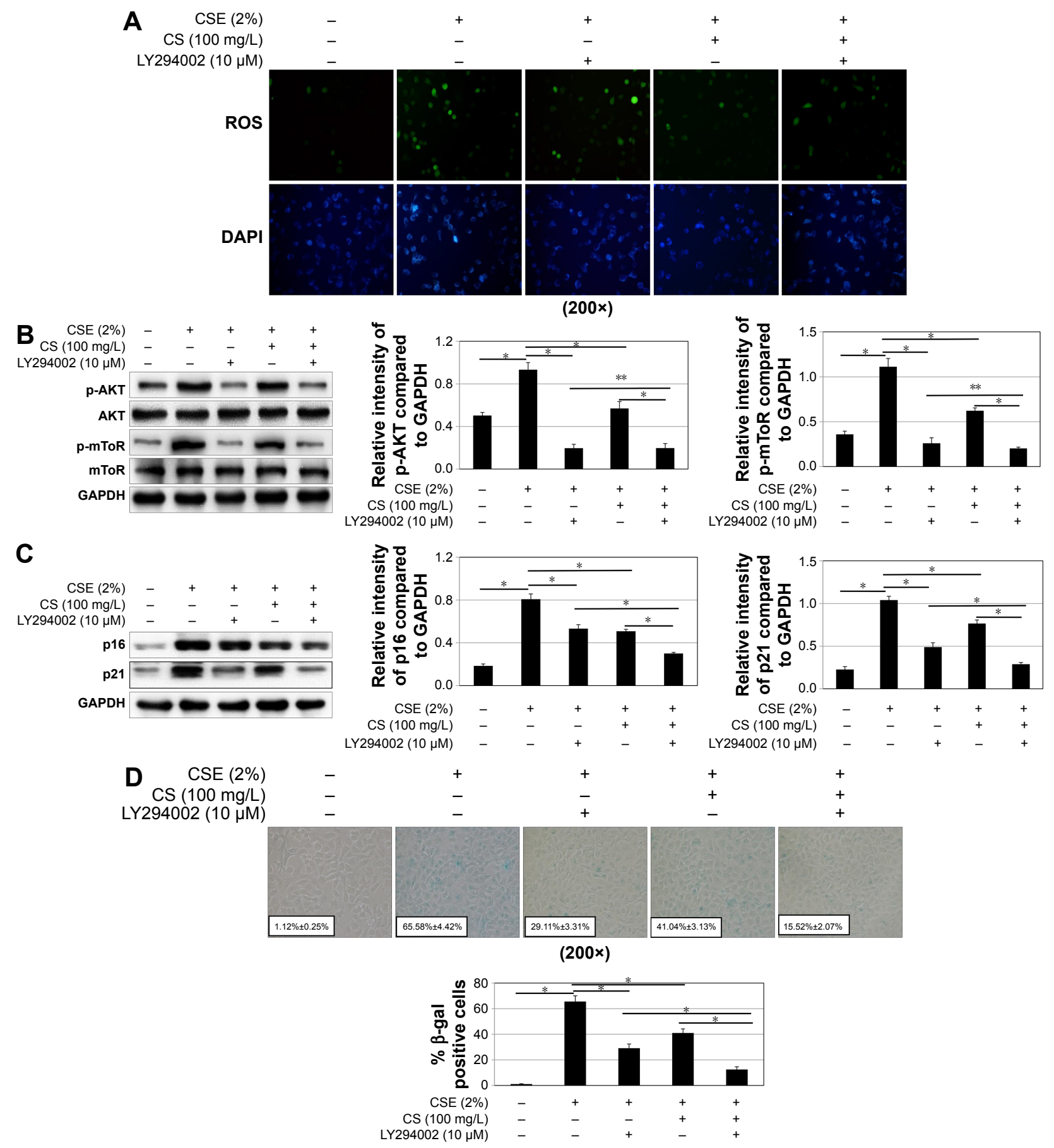

Figure 7 The effect of mTOR pathway activation and cellular senescence by blocking PI3K. 16HBE cells were stimulated with $2 \%$ CSE, 100 mg/L Cordyceps sinensis or/and $10 \mu$ M Ly294002.

Notes: (A) 16HBE cells were stained with ROS fluorescence. (B) Total protein was collected to detect the expressions of AKT, p-AKT, mTOR, and p-mTOR by Western blotting $\left({ }^{*} P<0.05\right.$, $\left.* * P>0.05\right)$. (C) The protein expressions of $p 16$ and $p 21$ were detected by Western blotting $\left({ }^{*} P<0.05\right)$. (D) The senescent cells were examined by $S A-\beta$-gal staining $(* P<0.05)$. Data are expressed as mean \pm SE. Results represent at least three independent experiments.

Abbreviations: CS, Cordyceps sinensis; CSE, cigarette smoke extract; DAPI, 4',6-diamidino-2-phenylindole; GAPDH, glyceraldehyde 3-phosphate dehydrogenase; HBE, human bronchial epithelial; mTOR, mammalian target of rapamycin; ROS, reactive oxygen species; SE, standard error.

mTOR signaling pathway, which leads to senescence. C. sinensis can block the process induced by CSE.

\section{Discussion}

Recently, it has been shown that cellular senescence might play a role in the pathogenesis of COPD. ${ }^{2}$ Cellular senescence is a process that results from a variety of stresses, leading to a state of irreversible growth arrest. ${ }^{27}$ Cellular senescence may be the same mechanism in many chronic diseases, ${ }^{28}$ including COPD.

The aim of current routine pharmacotherapy for stable COPD is to control disease progression by using 
bronchodilators and anti-inflammatory drugs; however, the treatment outcomes remain less than satisfactory. ${ }^{29}$ Treatment with Chinese herbal medicine is currently considered a complementary or alternative treatment and is being increasingly accepted worldwide. ${ }^{30,31} \mathrm{C}$. sinensis is one of the effective Chinese herbal medicines.

C. sinensis is a rare and precious medicinal mushroom having great medicinal value. Treatment with $C$. sinensis appreciably attenuated hypoxia-induced ROS generation, oxidation of lipids and proteins, and maintained antioxidant status. ${ }^{12}$ Current evidence suggested that $C$. sinensis was effective in treating COPD. ${ }^{32}$

Cellular senescence in COPD has a close association with smoking. Cigarette smoke exposure, the known main cause of COPD, ${ }^{33}$ has been widely demonstrated to accelerate cellular senescence. ${ }^{34,35}$ We proved that CSE could upregulate the level of senescence in human bronchial epithelial cells, the same as in previous studies.

Purported effects of the $C$. sinensis suggested a wide range of biological functions, such as use as an aphrodisiac, analgesic, and immune modulator and free radical scavenger. ${ }^{36}$ In recent years, $C$. sinensis has been investigated in animals and in vitro studies for antiaging effects. ${ }^{37}$ Similarly, we proved that $C$. sinensis could weaken the CSE-induced senescence. $C$. sinensis may be a useful method to resist senescence in patients with COPD.

The correlation between CSE and $C$. sinensis is not clear. There was research about genetic damage induced by cigarette smoke and the protective effects of the C. sinensis. ${ }^{38}$ Through our research, it was proven that CSE and $C$. sinensis are associated with cellular senescence.

Although the precise mechanisms of cellular senescence are still obscure, "the free radical theory of aging" has thus far been widely accepted. ${ }^{39}$ Cellular senescence has close association with chronic oxidative stress which originated from smoke. ${ }^{40}$ Under oxidative stress, the ROS increase. ${ }^{26}$ ROS is a series of derivatives that are generated during the metabolic process by aerobic cells, and participate in and influence a range of cellular signaling pathways, including activating PI3K/AKT/mTOR signaling pathway. ${ }^{41}$

There is increasing evidence that $\mathrm{PI} 3 \mathrm{~K} / \mathrm{AKT} / \mathrm{mTOR}$ signaling pathway is of critical importance in cellular senescence and aging, and inhibition of this pathway may extend the lifespan of many species. ${ }^{42,43} \mathrm{Xu}$ et $\mathrm{al}^{42}$ reported that active $\mathrm{AKT}$ inhibits the transcriptional activity of FOXO3a and thereby downregulates MnSOD, leading to an increased level of ROS that induced activation of the p53/p21 pathway and senescence. Senescent cells secrete cytokines and chemokines, known as the senescence-activated secretory phenotype, which amplifies and spreads cellular senescence. ${ }^{44}$ This suggests that activation of the mTOR pathway may play an important role in multimorbidity and inhibition of this pathway offers a future therapeutic opportunity. ${ }^{7}$

We detected ROS and the expressions of phospho-AKT and phospho-mTOR to evaluate the activation of ROS and $\mathrm{PI} 3 \mathrm{~K} / \mathrm{AKT} / \mathrm{mTOR}$ signaling pathway. Meanwhile, we found there was the same trend between the activation of ROS/ $\mathrm{PI} 3 \mathrm{~K} / \mathrm{AKT} / \mathrm{mTOR}$ signaling pathway and the increased senescence. Then, we blocked ROS and PI3K, and found the same weakening on cellular senescence. So we confirmed that $\mathrm{ROS}$ and $\mathrm{PI} 3 \mathrm{~K} / \mathrm{AKT} / \mathrm{mTOR}$ pathway was one of the important mechanisms of cellular senescence. We also found that there were still significant differences between CSE group and $\mathrm{CSE}+C$. sinensis group in activation of $\mathrm{mTOR}$ signaling pathway and senescence after blocking ROS; there were significant differences between CSE group and CSE + C. sinensis group in senescence after blocking PIK3.

The mechanism of cigarette smoke exposure resulting in cellular senescence mostly focused on excessive ROS production, ${ }^{45}$ mitochondrial damage, telomere shortening, p53, and p16-retinoblastoma protein pathways. ${ }^{46} \mathrm{We}$ confirmed that CSE can affect ROS generation, as reported earlier. At the same time, we investigated the connection between CSE and PI3K/AKT/mTOR signaling pathway. We found that CSE could affect the activation of mTOR signaling pathway, by testing the phosphorylation AKT and mTOR; blocking PI3K had influence on the senescence induced by CSE. We believe that the PI3K/AKT/mTOR signaling pathway is involved in the mechanism of CSEinduced senescence.

Our study had confirmed the effect of $C$. sinensis on senescence. But the specific mechanism is not clear. Zou et $\mathrm{al}^{12}$ found the inhibition effect of $C$. sinensis on oxidative stress. Park et $\mathrm{al}^{47}$ reported Cordyceps militaris extract might have protective effects against oxidative stress-induced premature senescence via scavenging ROS. In our study, we found that $C$. sinensis could affect ROS, which was consistent with previous studies. We also explored the influence of $C$. sinensis on the activation of mTOR signaling pathway, blocking ROS and PI3K could affect the function of $C$. sinensis. Antisenescence of $C$. sinensis may have close association with ROS and PI3K/ AKT/mTOR signaling pathway.

Although ROS and PI3K/AKT/mTOR signaling pathway play important roles in CSE-induced senescence, there were no specific quantified standards for us to find the particular points CSE and C. sinensis worked on. So, future studies need to be performed. 
This study demonstrated that exposure to CSE-induced cellular senescence, which can be reduced by $C$. sinensis. The CSE induces senescence via the ROS and PI3K/AKT/mTOR signaling pathway, and $C$. sinensis can weaken the pathway, which then reduces the CSE-induced cellular senescence. Our research provided a novel strategy for slowing the human aging process and aging-related diseases.

\section{Acknowledgments}

This research is supported by the National Nature Science Foundation of China (NSFC) (NSFC for LD, Grant number: 81270072) and the Natural Science Funding committee of Shandong province (SDNSF) (Grant number: ZR2015PH022). The funders had no roles in study design, data collection and analysis, decision to publish, and preparation of the manuscript.

\section{Disclosure}

The authors report no conflicts of interest in this work.

\section{References}

1. Global Initiative for Chronic Obstructive Lung Disease (GOLD). Global strategy for the diagnosis, management, and prevention of chronic obstructive pulmonary disease. Updated 2014. Available from: http://www.goldcopd.org/uploads/users/files/GOLD_Report_2014.pdf. Accessed April 16, 2014.

2. Kumar M, Seeger W, Voswinckel R. Senescence-associated secretory phenotype and its possible role in chronic obstructive pulmonary disease. Am J Respir Cell Mol Biol. 2014;51(3):323-333.

3. Ziegler DV, Wiley CD, Velarde MC. Mitochondrial effectors of cellular senescence: beyond the free radical theory of aging. Aging Cell. 2015;14(1):1-7.

4. Manuel C, Blasco MA, Manuel S. Cellular senescence in cancer and aging. Cell. 2007;130(2):223-233.

5. Barnes PJ. Mechanisms of development of multimorbidity in the elderly. Eur Respir J. 2015;45(3):790-806.

6. Johnson SC, Rabinovitch PS, Kaeberlein M. mTOR is a key modulator of ageing and age-related disease. Nature. 2013;493(7432):338-345.

7. Lamming DW, Ye L, Sabatini DM, Baur JA. Rapalogs and mTOR inhibitors as anti-aging therapeutics. J Clin Invest. 2013;123(3):980-989.

8. Li JS, Li SY, Yu XQ, et al. Bu-Fei Yi-Shen granule combined with acupoint sticking therapy in patients with stable chronic obstructive pulmonary disease: a randomized, double-blind, double-dummy, active-controlled, 4-center study. $J$ Ethnopharmacol. 2012;141(2): 584-591.

9. Li SY, Li JS, Wang MH, et al. Effects of comprehensive therapy based on traditional Chinese medicine patterns in stable chronic obstructive pulmonary disease: a four-center, open-label, randomized, controlled study. BMC Complement Altern Med. 2012;12:197.

10. Liu Y, Wang J, Wang W, Zhang H, Zhang X, Han C. The chemical constituents and pharmacological actions of Cordyceps sinensis. Evid Based Complement Alternat Med. 2015;2015:575063.

11. Dong CH, Yao YJ. In vitro evaluation of antioxidant activities of aqueous extracts from natural and cultured mycelia of Cordyceps sinensis. Food Sci Technol. 2008;41(4):669-677.

12. Zou Y, Liu Y, Ruan M, et al. Cordyceps sinensis oral liquid prolongs the lifespan of the fruit fly, Drosophila melanogaster, by inhibiting oxidative stress. Int J Mol Med. 2015;36(4):939-946.
13. Singh M, Tulsawani R, Koganti P, Chauhan A, Manickam M, Misra K. Cordyceps sinensis increases hypoxia tolerance by inducing heme oxygenase-1 and metallothionein via Nrf2 activation in human lung epithelial cells. Biomed Res Int. 2013;2013:569206.

14. Kirkham PA, Barnes PJ. Oxidative stress in COPD. Chest. 2013;144(1): 266-273.

15. Carp H, Janoff A. Possible mechanisms of emphysema in smokers. In vitro suppression of serum elastase-inhibitory capacity by fresh cigarette smoke and its prevention by antioxidants. Am Rev Respir Dis. 1978;118(3):617-621.

16. Cui M, Guo XH, Li H. Cordyceps sinensis extractant protect against cyclosporine induced injury of tubular epithelium cell. J Nephrol Daily Transplant. 2004;13(1):44-48.

17. Ma LL, Yang XY, Gao JZ. Experimental study on effect of Bailing Capsule on dendritic cells in mice. Zhong Guo Zhong Xi Yi Jie He Za Zhi. 2007;27(10):905-908.

18. Badinloo M, Esmaeili-Mahani S. Phosphatidylinositol 3-kinases inhibitor LY294002 potentiates the cytotoxic effects of doxorubicin, vincristine, and etoposide in a panel of cancer cell lines. Fundam Clin Pharmacol. 2014;28(4):414-422.

19. Chen S, Ren Q, Zhang J, et al. N-acetyl-L-cysteine protects against cadmium-induced neuronal apoptosis by inhibiting ROS-dependent activation of Akt/mTOR pathway in mouse brain. Neuropathol Appl Neurobiol. 2014;40(6):759-777.

20. Wu J, Dong F, Wang RA, et al. Central role of cellular senescence in TSLP-induced airway remodeling in asthma. PLoS One. 2013;8(10): e77795.

21. Zhao JP, Jiao XA, Wu JX, et al. FIZZ1 promotes airway remodeling in asthma through the PTEN signaling pathway. Inflammation. 2015; 38(4):1464-1472.

22. Kuilman T, Michaloglou C, Vredeveld LC, et al. Oncogene-induced senescence relayed by an interleukin-dependent inflammatory network. Cell. 2008;133(6):1019-1031.

23. Tuder RM, Yoshida T. Stress responses affecting homeostasis of the alveolar capillary unit. Proc Am Thorac Soc. 2011;8(6):485-491.

24. Campisi J, d'Adda di Fagagna F. Cellular senescence: when bad things happen to good cells. Nat Rev Mol Cell Biol. 2007;8(9): 729-740.

25. Dimri GP, Lee X, Basile G, et al. A biomarker that identifies senescent human cells in culture and in aging skin in vivo. Proc Natl Acad Sci U S A.1995;92(20):9363-9367.

26. Schieber M, Chandel NS. ROS function in redox signaling and oxidative stress. Curr Biol. 2014;24(10):R453-R462.

27. Nyunoya T, Monick MM, Klingelhutz A, Yarovinsky TO, Cagley JR, Hunninghake GW. Cigarette smoke induces cellular senescence. Am J Respir Cell Mol Biol. 2006;35(6):681-688.

28. Fabbri E, Zoli M, Gonzalez-Freire M, Salive ME, Studenski SA, Ferrucci L. Aging and multimorbidity: New tasks, priorities, and frontiers for integrated gerontological and clinical research. $J$ Am Med Dir Assoc. 2015;16(8):640-647.

29. Qaseem A, Wilt TJ, Weinberger SE, et al. Diagnosis and management of stable chronic obstructive pulmonary disease: a clinical practice guideline update from the American College of Physicians, American College of Chest Physicians, American Thoracic Society, and European Respiratory Society. Ann Intern Med. 2011;155(3): 179-191.

30. Chen X, May B, Di YM, et al. Oral Chinese herbal medicine combined with pharmacotherapy for stable COPD: A systematic review of effect on BODE index and six minute walk test. PLoS One. 2014;9(3): e91830.

31. Hu J, Zhang J, Zhao W, Zhang Y, Zhang L, Shang H. Cochrane systematic reviews of Chinese herbal medicines: an overview. PLoS One. 2011;6(12):e28696.

32. Wei M, Song YL, Zhang S, Zhang L, Min FU. Cordyceps sinensis for chronic obstructive pulmonary diseases: a systematic review. Chin $J$ Evidence-Based Med. 2013;13(11):1373-1381. 
33. Eisner MD, Anthonisen N, Coultas D, et al. An official American Thoracic Society public policy statement: Novel risk factors and the global burden of chronic obstructive pulmonary disease. Am J Respir Crit Care Med. 2010;182(5):693-718.

34. Hara H, Araya J, Takasaka N, et al. Involvement of creatine kinase B in cigarette smoke-induced bronchial epithelial cell senescence. Am J Respir Cell Mol Biol. 2012;46(3):306-312.

35. Fujii S, Hara H, Araya J, et al. Insufficient autophagy promotes bronchial epithelial cell senescence in chronic obstructive pulmonary disease. Oncoimmunology. 2012;1(5):630-641.

36. Yamaguchi Y, Kagota S, Nakamura K, Shinozuka K, Kunitomo M. Antioxidant activity of the extracts from fruiting bodies of cultured Cordyceps sinensis. Phytother Res. 2000;14(8):647-649.

37. Ji DB, Ye J, Li CL, Wang YH, Zhao J, Cai SQ. Antiaging effect of Cordyceps sinensis extract. Phytother Res. 2009;23(1):116-122.

38. Ma J, Bi S. Protective effects of Cordyceps sinensis (Berk) Sacc, artificial pypha on genetic damage induced by cigarette smoke. Beijing $D a$ Xие Xие Bao. Zi ran ke xue bao. 1996;32(4):521-526.

39. Harman D. Free radical theory of aging: an update: increasing the functional life span. Ann NY Acad Sci. 2006;1067:10-21.

40. Bono R, Tassinari R, Bellisario V, et al. Urban air and tobacco smoke as conditions that increase the risk of oxidative stress and respiratory response in youth. Environ Res. 2015;137:141-146.
41. Jin SY, Lee HS, Kim EK, Ha JM, Kim YW, Bae S. Reactive oxygen species and PI3K/Akt signaling in cancer. Free Radic Biol Med. 2014; 75(Suppl 1):S34-S35.

42. Xu Y, Li N, Xiang R, Sun P. Emerging roles of the p38 MAPK and $\mathrm{PI} 3 \mathrm{~K} / \mathrm{AKT} / \mathrm{mTOR}$ pathways in oncogene-induced senescence. Trends Biochem Sci. 2014;39(6):268-276.

43. Harrison DE, Strong R, Sharp ZD, et al. Rapamycin fed late in life extends lifespan in genetically heterogeneous mice. Nature. 2009; 460(7253):392-395.

44. Salama R, Sadaie M, Hoare M, Narita M. Cellular senescence and its effector programs. Genes Dev. 2014;28(2):99-114.

45. Ito S, Araya J, Kurita Y, et al. PARK2-mediated mitophagy is involved in regulation of HBEC senescence in COPD pathogenesis. Autophagy. 2015;11(3):547-559.

46. Hara H, Araya J, Ito S, et al. Mitochondrial fragmentation in cigarette smoke-induced bronchial epithelial cell senescence. Am J Physiol Lung Cell Mol Physiol. 2013;305(10):L737-L746.

47. Park JM, Lee JS, Lee KR, Ha SJ, Hong EK. Cordyceps militaris extract protects human dermal fibroblasts against oxidative stress-induced apoptosis and premature senescence. Nutrients. 2014;6(9):3711-3726.
International Journal of COPD

\section{Publish your work in this journal}

The International Journal of COPD is an international, peer-reviewed journal of therapeutics and pharmacology focusing on concise rapid reporting of clinical studies and reviews in COPD. Special focus is given to the pathophysiological processes underlying the disease, intervention programs, patient focused education, and self management protocols.

\section{Dovepress}

This journal is indexed on PubMed Central, MedLine and CAS. The manuscript management system is completely online and includes a very quick and fair peer-review system, which is all easy to use. Visit http://www.dovepress.com/testimonials.php to read real quotes from published authors. 